\title{
Impfethik und das Recht auf größtmögliche Gesundheit
}

\section{Vaccinations Ethics and Health Care}

Eine Arbeitstagung am 11./12. Oktober 2012 an der Ruhr-Universität Bochum zur Impfethik behandelte ein gesundheitspolitisch äußerst wichtiges und als Folge neuer Aspekte immer wieder zu diskutierendes Thema der Public Health Ethik [9]; deshalb wird hierüber auf den Seiten 50-52 berichtet [3]. Es besteht allgemeiner Konsens darin, dass die einzige Möglichkeit zur Bewältigung der absehbaren Probleme in der weltweiten Gesundheitsversorgung die Prävention darstellt. Unbestritten ist auch, dass eine der wichtigsten Maßnahmen zur Vermeidung schwerwiegender Krankheiten die anerkannten und z.B. von der STIKO empfohlenen Impfungen darstellen. In § 24 der UN-Kinderrechtskonvention von 2002 wird allen Kindern das Recht auf größtmögliche Gesundheit zugestanden, gleichzeitig haben UN und UNICEF verkündet, dass jedes Kind ein Recht auf Impfung gegen verhütbare Krankheiten hat [1].

Eindrucksvoll wird in einem jüngst im Lancet erschienenen Bericht über die aktuellen Ursachen der Mortalität von Kindern zwischen 0 und 5 Jahren weltweit gezeigt, dass hierfür neben perinatalen Komplikationen vor allem Pneumonien verantwortlich sind, die durch die Pneumokokken-Impfungen deutlich reduziert werden können [5]. Praktisch alle schwerwiegenden Impfkomplikationen, die bis heute diskutiert werden, sind in umfangreichen, gut dokumentierten Studien als Gefährdungspotenzial im Vergleich zu den Wildinfektionen eindrucksvoll widerlegt worden [7]. So konnte z. B. bei einer großen Zahl von Menschen, bei denen wegen einer Entwicklungsstörung mit Epilepsie nach einer Keuchhusten-Impfung ein Impfschaden anerkannt worden war, retrospektiv ein Dravet-Syndrom - eine genetisch bedingte Enzephalopathie - festgestellt werden [6].

Die zeitgerechte Durchführung der Impfungen, vor allem gegen Masern, Mumps und Röteln hängt viel von der ärztlichen Beratung ab; eine Verunsicherung der Eltern durch alternativ eingestellte Ärzte kann sich nachteilig auswirken [8]. Aber selbst bei Frühgeborenen mit einem Geburtsgewicht unter $1500 \mathrm{~g}$ sind die üblichen Routineimpfungen ab dem 3. Lebensmonat ohne wesentliche Komplikationen durchführbar [10]. Nur bei den seltenen schweren kombinierten Immundefekten (SCID) oder primären Antikörpermangelerkrankungen ist eine Lebendimpfung kontraindiziert, was in den entsprechenden Leitlinien hervorgehoben wird $[2,4]$.

Leider gibt es unverändert in Deutschland zu viele Menschen, die entweder aus grundsätzlichen Erwägungen oder aus mangelndem Interesse die empfohlenen Impfungen bei ihren Kindern und bei sich selbst nicht durchführen lassen. Besonders problematisch ist in diesem Zusammenhang die Einstellung einzelner Ärzte und Hebammen, die einen großen Einfluss gerade auf junge Eltern haben. Impfverweigerung einerseits und der Wegfall von Grenzen sowie die Migration andererseits führen immer wieder zum Auftreten von Infektionskrankheiten in Deutschland. Dies belegen die zunehmenden Masernausbrüche, aber auch das Vorkommen von Diphtherie und Keuchhusten in den vergangenen Jahren in erschreckender Weise. Verhindert würden diese Ausbrüche durch einen entsprechenden Impfschutz, da dann die Erreger nicht weitergegeben werden. Von den großen kinderärztlichen Organisationen, z.B. der Deutschen Gesellschaft für Kinder- und Jugendmedizin, dem Berufsverband der Kinder- und Jugendärzte, der Deutsche Gesellschaft für Pädiatrische Infektiologie und der Deutschen Gesellschaft für Sozialpädiatrie und Jugendmedizin ist daher wiederholt gefordert worden, die Aufnahme in eine Kindergemeinschaftseinrichtung/Kindertageseinrichtung von einem ausreichenden Impfschutz nach den Empfehlungen der STIKO abhängig zu machen, sofern keine individuellen Kontraindikationen vorliegen. Dies wird derzeit aber von allen politischen Parteien abgelehnt.

Betont wird die Bedeutung der Öffentlichkeitsarbeit zum Impfen als wichtige präventive Maßnahme und die Notwendigkeit eines allgemeingültigen nationalen Impfplans. Diese und weitere ethische Aspekte zum Impfen im Spannungsfeld zwischen Individuum und Gesellschaft haben ein ständiges Thema der Public-Health-Forschung zu sein. Der Beitrag zur Impfethik spricht viele dieser Fragen an, die gerade auch in den kommenden Monaten immer wieder auf die Agenda der öffentlichen Diskussionen in Deutschland zu setzen sind.

H. M. Straßburg

Präsident der Deutschen Gesellschaft für Sozialpädiatrie und Jugendmedizin

\section{Danksagung}

$\nabla$

Frau Dr. R. Klein, Saarbrücken, danke ich für die Beratung bei der Abfassung des Statements.

Literatur

1 Faden R, Shebaya S. Public Health Ethics, - the Stanford Encyclopaedia of Philosophy (Summer 2010 Edition), Editor E.N. Zaita, 2010

2 Farmand $S$, Baumann $U$, von Bernuth $H$ et al. Interdisziplinäre AWMF-Leitlinie zur Diagnostik von primären Immundefekten (S2k). Klin Padiatr 2011; 223: $378-385$ 
3 Kries von R. Impfethik: Bericht über eine Arbeitstagung an der Ruhr - Universität Bochum. Klin Padiatr 2013; 225: 50-52

4 Krudewig J, Baumann $U$, Bernuth von $\mathrm{H}$ et al. Interdisziplinäre AWMFLeitlinie zur Therapie primärer Antikörpermangelerkrankungen. Klin Padiatr 2012; 224: 404-415

5 Liu L, Johnson HL, Cousens S et al. Global, regional, and national causes of child mortality: an updated systematic analysis for 2010 with time trends since 2000. Lancet 2012; 379: 2151-2161

6 McIntosh AM, McMahon J, Dibbens LM et al. Effects of Vaccination on onset and outcome of Dravet-syndrome: A retrospective study. The Lancet Neurology 2010; 9: 595-598
7 Schneeweiß B, Pfleiderer M, Keller-Stanislawski B. Impfsicherheit heute. Dtsch Arztebl 2008; 105: 590-595

8 Schönberger K, Ludwig MS, Wildner M et al. Timely MMR vaccination in infancy: Influence of attitudes and medical advice on the willingness to vaccinate. Klin Padiatr 2012; 224: 1-7

9 Vollmann J. International Symposium „Vaccination Ethics“ 11./12. October 2012, Bochum

10 Ziegler B, Straßburg HM. Impfstatus bei Frühgeborenen mit einem Geburtsgewicht unter $1500 \mathrm{~g}$ im Alter von 2 Jahren - eine deutschlandweite Studie. Klin Padiatr 2010; 222: 243-247 